\title{
Strategy and Enhanced Temperature Determination in a Laser Heated Diamond Anvil Cell
}

\section{Citation}

Deemyad, Shanti, Anthony N. Papathanassiou, and Isaac F. Silvera. 2009. Strategy and enhanced temperature determination in a laser heated diamond anvil cell. Journal of Applied Physics 105(9): 093543.

\section{Published Version}

doi:10.1063/1.3117517

\section{Permanent link}

http://nrs.harvard.edu/urn-3:HUL.InstRepos:8944724

\section{Terms of Use}

This article was downloaded from Harvard University's DASH repository, and is made available under the terms and conditions applicable to Open Access Policy Articles, as set forth at http:// nrs.harvard.edu/urn-3:HUL.InstRepos:dash.current.terms-of-use\#OAP

\section{Share Your Story}

The Harvard community has made this article openly available.

Please share how this access benefits you. Submit a story.

\section{Accessibility}




\title{
Strategy and Enhanced Temperature Determination in a Laser Heated Diamond Anvil Cell
}

\author{
Shanti Deemyad, Anthony N. Papathanassiou, and Isaac F. Silvera \\ Lyman Laboratory of Physics, Harvard University, Cambridge MA 02138
}

We show that a strategy for increased accuracy in temperature determination by optical pyrometry when the wavelength dependence of the emissivity is unknown is to measure the spectral irradiance at short wavelengths. We then introduce an improved method of determining the temperature in laser heated diamond anvil cells. In general a blackbody source is used to determine the optical transfer function required for determining the blackbody curve. By using the thermal radiation of a heated absorber at ambient pressure and known temperature, uncertainties in the temperature determination caused by the wavelength dependence of the emissivity of the heated absorber can be eliminated. Temperature determination reduces to a one-parameter fit to the blackbody curve rather than the usual two parameters (emissivity and temperature), leading to increased precision and accuracy. 


\section{Introduction}

Laser heating in diamond anvil cells (DACs) is an important technique that allows the development of high temperature conditions at static high pressure and has been widely used in many disciplines [1]. In a laser heated diamond anvil cell (LHDAC) pressures of the order of $100 \mathrm{GPa}$ and temperatures of several thousand Kelvin can be achieved. In condensed matter physics, chemistry, material science, and earth and planetary sciences, the LHDAC enables mapping of P-V-T phase diagrams, discovery and study of high P-T phases, melting lines, and studying the electronic, magnetic, and structural properties of materials at elevated temperatures and pressures $[2,3]$. In earth and planetary sciences studies under such conditions are crucial in understanding the interior of planets [4].

Pressure and temperature are the two most important parameters to be determined in LHDAC experiments. Advances in the LHDAC technology have continued, e.g., [5-9], since its inception [10]; still the complexity of these measurements can lead to significant uncertainty in determining pressure and temperature. The most commonly used method for determining the temperature of a sample in an LHDAC is spectral optical pyrometry [11]. In this paper we consider the strategy for optimally determining temperature and a present a technique that can improve the accuracy and precision in temperature determination. The advantage of this technique is demonstrated by experimental measurements using platinum to absorb the laser power.

\section{Temperature Measurement in the LHDAC: Theory}

Optical pyrometry in an LHDAC allows non-contact, remote temperature determination of a material. The laser beam is focused on a sample or an absorber that heats to a local 
thermodynamic equilibrium temperature described by the Planck radiation law and it is the temperature of the absorber that is determined. For temperature measurement by optical pyrometry the thermal irradiance from the absorber is collected as a function of wavelength; temperature is determined by fitting to the Planck thermal radiation function

$$
I(\lambda, T) \Delta \lambda=\frac{2 h c^{2}}{\lambda^{5}} \cdot \frac{\varepsilon(\lambda, T)}{\exp (h c / \lambda k T)-1} \Delta \lambda \equiv \varepsilon(\lambda, T) I_{\lambda, T}^{b b} \Delta \lambda
$$

Here, $I(\lambda, T)$ is the spectral irradiance, $\lambda$ the wavelength, $T$ the temperature, $\varepsilon(\lambda, T)$ the emissivity, $h$ Planck's constant, $c$ the speed of light, and $k$ the Boltzmann constant. The emissivity of a blackbody is 1 , while an absorber is at best a graybody with wavelength independent emissivity less than 1 . In reality the emissivity usually is both wavelength and temperature dependent. In order to accurately determine the temperature, the BB lineshape, which is usually distorted by the measurement instruments, must be determined and the emissivity of the absorber must be known as a function of wavelength and temperature. It is sufficient to measure the irradiance in a limited spectral region and fit to the BB curve in that region. If the temperature is not uniform in space and time (for example in pulsed laser heating), then appropriate corrections must be made [12]. For purposes of discussion we introduce the notation $I_{\lambda, T}^{b b} \equiv B B(\lambda, T)$ for a blackbody emitter and $G B \equiv \varepsilon I_{\lambda, T}^{b b}$, with $\varepsilon=$ constant for a graybody emitter.

\section{Strategies for Determining the Temperature}

Ideally to determine the temperature by pyrometric methods one would like to measure the irradiance over a broad spectral range, but this is impractical and unnecessary. In some instruments, spectral measurements are made at only one or two wavelengths and in principle, accurate temperatures can be determined, although measuring at several wavelengths should 
increase the accuracy of the measurement. One of the most egregious problems is the lack of knowledge of the wavelength and temperature dependence of the emissivity (in this section we concentrate the discussion on the wavelength dependence). When knowledge of the wavelength dependence of the emissivity is lacking, temperatures in an LHDAC are usually determined by fitting the irradiance to a GB curve. This can lead to serious errors in the accuracy, as we shall demonstrate.

In Fig. 1 we multiply the blackbody curve for $\mathrm{T}=1000 \mathrm{~K}$ by the experimental emissivity of platinum to show how wavelength dependent emissivity can affect the BB curve. One sees that the peak of the platinum curve is shifted to shorter wavelength. Both curves look like BB curves and if Wien's Law ( $\lambda_{\max } T=$ constant $)$ is used to determine the temperature for the platinum curve, $\mathrm{T}=1160 \mathrm{~K}$ is found, rather than the true temperature of $1000 \mathrm{~K}$ (Note that Wien's constant is independent of the absolute value of the emissivity if the emitter is a graybody). Alternately, if the wavelength dependence of the emissivity is unknown and the irradiance is fit to a GB curve, shown in Fig. 1, one finds a serious error in the temperature, with $T=1184 \pm 0.5 \mathrm{~K}$ and $\varepsilon=0.07 \pm 0.000$, resulting in an inaccuracy of $184 \mathrm{~K}$ or $18 \%$. For this fit we used 6000 points in the wavelength range $0.5-6.5$ micron with no noise added to the irradiance values.

One strategy for improving the accuracy is to select the wavelength region for the fit. In an LHDAC one is interested in the fitted value of $\mathrm{T}$, whereas the emissivity (or emittance) is less significant, and does not represent a reliable measurement value. Because of the nature of the $\mathrm{BB}$ curve, exponential in $\mathrm{T}$ for short wavelengths and linear in $\mathrm{T}$ for long wavelengths, we expect the impact of the wavelength dependence of the emissivity on the accuracy of the temperature determination to be much less if irradiance is measured and fitting is done in the 
exponential part of the curve. In Table I we show the results for fitting in several different wavelength regions using a graybody approximation when the emissivity is actually wavelength dependent, confirming our conjecture.

To quantify this we have defined a parameter $\Lambda$ that represents the normalized difference between a graybody curve (fitting curve) and a non graybody emitter curve, with $\varepsilon_{N G B}(\lambda)=0.33235-0.047064 \lambda[13]$ and $\mathrm{T}=1000$, in a given wavelength interval $(\lambda, \lambda+\Delta \lambda)$ :

$$
\Lambda(\varepsilon, T)=\sum_{\lambda}^{\lambda+\Delta \lambda} \frac{\left[\varepsilon_{N G B}(\lambda) B B_{T=1000 K}-G B(\varepsilon, T)\right]^{2}}{\left[\varepsilon_{N G B}(\lambda) B B_{T=1000 K}+G B(\varepsilon, T)\right]^{2}} .
$$

The best fit value(s) of $\varepsilon, T$ for the GB curve minimizes $\Lambda$. In Fig. 2 we show the results for $\Lambda$ as a function of $\mathrm{T}$ and $\varepsilon$. Fitting in the short wavelength region a global minimum is found (not visible in the figure) and there is a relatively narrow range of values for T. Fitting only to the longer wavelength region, the global minimum is very shallow demonstrating the possibility of a large inaccuracy in determining $\mathrm{T}$, in particular if noise is added to the data points used in the fit. The situation is even more extreme if the fit is done in the long wavelength region, beyond the peak.

\section{An Improved Method and Optical Transfer Functions in the LHDAC}

For practical reasons, the optimum wavelength region where the irradiance of a non blackbody emitter is collected for temperature determination cannot always be chosen in the exponential part of the thermal radiation curve. More specifically at lower temperatures in an LHDAC ( $T \sim 700 K$ or lower) there are very few thermally radiated photons at shorter wavelengths and for improved signal-to-noise and precision, measurements should be made closer to the BB peak where uncertainties in wavelength dependence of emissivity can give rise to large errors in temperature. In principle use of an absorber with well-known emissivity would eliminate this problem, however this would create an experimental limitation in the choice of an 
absorber. In this section of the paper we introduce a technique in optical pyrometry that removes the uncertainties in temperature determination due to the lack of knowledge of the wavelength dependence of the emissivity without direct measurement of the wavelength dependence of emissivity. This technique can be used for any material that is chosen as the absorber and thermal emitter in an LHDAC.

In general, for a BB source, the optical response of a system, including detector, spectrometer, filters, etc, is wavelength dependent so that the measured irradiance is distorted from the BB curve and must be corrected. The correction can be made by determining the response using a calibrated source of radiation such as a BB source at a known temperature. This response is usually characterized by the optical transfer function, $T F_{\lambda}^{b b}$. In principle, the transfer function is independent of the temperature of the BB source and is defined as

$$
T F_{\lambda}^{b b}=\frac{I_{\lambda, T}^{b b}}{S_{\lambda, T}^{b b}},
$$

where $I_{\lambda, T}^{b b}$ is the theoretical (calculated) Planck BB irradiance at temperature $T$ and wavelength $\lambda$, and $S_{\lambda, T}^{b b}$ is the signal of the BB source collected by the optical system at temperature $T$ and wavelength $\lambda$. There is always a finite spectral width or resolution, $\delta \lambda$, over which the signal is measured. The transfer function is independent of the temperature only if $\delta \lambda$ is small enough so that the optical response of the system is not a rapidly varying function over the resolution width and the average signal over $\delta \lambda$ is a good approximation to the signal at $\lambda$.

The spectral irradiance of the absorber at any other unknown temperature $T_{x}$ can be determined from the following relation,

$$
I_{\lambda, T_{x}}^{a b s o r b e r}=T F_{\lambda}^{b b} \times S_{\lambda, T_{x}}^{a b s o r b e r}
$$


which corrects the measured signal for temperature $T_{x}$ to an undistorted irradiance of the absorber at $T_{x}$.

Here, $I_{\lambda, T_{x}}^{a b s o r b e r}$ and $S_{\lambda, T_{x}}^{a b s o r b e r}$ are the irradiance and the signal of the absorber at $\lambda$ and $T_{x}$, respectively, and

$$
I_{\lambda, T_{x}}^{a b s o r b e r}=\varepsilon\left(\lambda, T_{x}\right)^{a b s o r b e r} \times I_{\lambda, T_{x}}^{b b}
$$

The unknown temperature, $T_{x}$, is determined by fitting the result of Eq. (4) to a Planckian spectrum. Since most absorbers are not blackbodies the emissivity of the absorber, $\varepsilon(\lambda, T)$, which includes its temperature and wavelength dependence should be determined to achieve high accuracy. However, the wavelength dependence of the emissivity for most materials is not known, and as mentioned earlier, in most cases emissivity is assumed to be independent of wavelength and its value is determined using a two-parameter $\left(\varepsilon, T_{x}\right)$ fit to the Planck function. This uncertainty in the wavelength dependence of the emissivity can lead to serious errors in the accuracy of the temperature, substantially larger than $10 \%$, while a two parameter fit leads to lower precision than fitting to a single parameter.

Here we introduce a method in which the emissivity of the sample is absorbed into the optical transfer function. We show that the unknown wavelength dependence of the emissivity will be removed by such a method and a more precise determination of the temperature is made using a one-parameter $(\mathrm{T})$ fit to the Planck blackbody radiation function.

Define the optical transfer function which includes the emissivity of the absorbing material, $T F_{\lambda}^{a b s o r b e r}$, as follows:

$$
T F_{\lambda}^{a b s o r b e r}=\frac{I_{\lambda, T}^{b b}}{S^{a b s o r b e r}},
$$


then

$$
T F_{\lambda}^{b b}=\varepsilon(\lambda)^{a b s o r b e r} \times T F_{\lambda}^{a b s o r b e r}
$$

From Eqs. 3-7, if we heat the absorber to an unknown temperature, $T_{x}$, the following relation will be true because the emissivity term that appears in $T F_{\lambda}^{a b s o r b e r}$ will be cancelled by the emissivity term in the numerator of $S_{\lambda, T_{x}}^{\text {absorber }}$,

$$
I_{\lambda, T_{x}}^{\text {absorber }}=T F_{\lambda}^{\text {absorber }} \times S_{\lambda, T_{x}}^{\text {absorber }}=B B(\lambda, T)
$$

Thus, the wavelength dependence of the emissivity is eliminated without knowledge of the absolute value of the emissivity of the absorber, without using a blackbody source. $T_{x}$ can be determined by fitting to the Planck blackbody radiation function, $B B(\lambda, T)$, with a single fit parameter.

Experimentally $T F_{\lambda}^{\text {absorber }}$ can be measured by using a sample that is identical to the absorber used in the LHDAC and heating it at ambient pressure to a known temperature $T$. However, here the temperature or pressure dependence of the emissivity is not taken into account. As long as the temperature or pressure dependence of the emissivity is weakly varying, $T F_{\lambda}^{\text {absorber }}$ gives a good first order approximation to the true value and provides a much more accurate temperature determination than found using a graybody fit. If the temperature dependence of the transfer function is known in the temperature interval of interest, the simplest approach to determine the temperature would be to normalize the Planck blackbody function by dividing it by $T F^{\text {absorber }}(\lambda, T)$, and determine the temperature of the absorber at any other unknown temperature by simply fitting the $S_{\lambda, T_{x}}^{\text {absorber }}$ to the normalized Planck function. We mention here that since the radiation from the absorber passes through the pressurization medium and one diamond in a DAC, a correction must be made for these effects. 


\section{Temperature Measurement in the LHDAC: Experiment}

Here we demonstrate how absorbing the emissivity into the transfer function can improve the accuracy of the temperature measurement. Figure 3 shows the schematic layout of our optical system. We measure in the near IR at several discrete wavelengths [14] in the wavelength range between $1.8-3.1 \mu \mathrm{m}$, using a series of 5 narrow band filters to determine the wavelength dependence of the irradiance of the sample (optical spectrometers and CCDs can be used for measuring in the visible). Thermal radiation from a platinum foil, embedded inside a homemade oven, is collected by a $\mathrm{CaF}_{2}$ lens with 1:1 magnification, focused into a 25 micron diameter pinhole which spatially filters the light. This light is then collected by two $\mathrm{CaF}_{2}$ lenses, and focused onto a liquid nitrogen cooled $\mathrm{InSb}$ detector. The temperature of the platinum is measured with an R type thermocouple that is attached to the surface of the platinum, and for this demonstration the temperature is increased in increments of $\sim 100 \mathrm{~K}$ between $625-1150 \mathrm{~K}$. The transfer function, $T F_{\lambda, T}^{a b s o r b e r}$ (shown in Fig. 4a, inset) was determined using Eq. 6, where $I_{\lambda, T}^{b b}$ was evaluated at temperature T, measured by the thermocouple. The signal from the platinum, $S_{\lambda, T}^{P t}$, was measured with the system in Fig. 3. We also measured $T F_{\lambda, T}^{b b}$ by replacing the Pt source with a homemade BB source with $\varepsilon \geq 0.99$. The temperature of the BB source was measured with an $\mathrm{R}$ type thermocouple with its tip imbedded inside the BB cavity. $T F_{\lambda, T}^{b b}$ was determined by calculating $I_{\lambda, T}^{b b}$ at a temperature determined by the thermocouple, and $S_{\lambda, T}^{b b}$ was the signal detected by the InSb detector.

To demonstrate the advantage of eliminating the emissivity, we determined the temperature of a Pt foil by two methods and compared the results to the temperature of the 
sample, measured independently with a thermocouple. We calculated both $T F_{\lambda, T}^{\text {absorber }}$ for a Pt absorber and $T F_{\lambda, T}^{b b}$. The temperature of the Pt absorber was then determined for five temperatures by using

a) Emissivity eliminated method with a one parameter fit (T).

b) Emissivity eliminated method with two fitting parameters ( $\mathrm{T}$ and $\varepsilon=$ const ; this assumes that the absolute value of the emissivity is not known).

c) Conventional correction of the signal by $T F_{\lambda, T}^{b b}$ and assuming graybody behavior.

The results are summarized in Table II and some of the fits are shown in Fig. 4 a, b. $T_{T C}$ is the temperature of the absorber measured directly by thermocouple. $T_{P t-1 P}$ is the temperature of the sample determined by using the one-parameter fit to Planck blackbody function and the signal of the sample was corrected for spectral distortion by $T F_{\lambda}^{a b s o r b e r} . T_{P t-2 P}$ and $\varepsilon_{P t-2 P}$ are the temperature and scaling factors of the Pt determined by the two-parameter fit to the Planck graybody function. Here the signal of the sample was also corrected by $T F_{\lambda}^{a b s o r b e r} . T_{b b-2 P}$ and $\varepsilon_{b b-2 P}$ are the temperature and emissivity of the platinum foil determined by using a twoparameter fit to the Planck graybody function in which the signal of the platinum was corrected by multiplying by $T F_{\lambda}^{b b}$. Note that in Fig. $4 \mathrm{a}$ and $\mathrm{b}$ the precision of the points fit to the curves for both cases is excellent, but there is a serious error in the accuracy in Fig. 4b. Fig. 5 summarizes important results by plotting the fitted temperatures against the temperature measured with a thermocouple.

We used the fitting routine of "Igor Pro 5.0" for data analysis. In curve fitting, Igor uses raw data and a function with unknown coefficients and chooses the coefficients such that the function matches the raw data to minimize the value of Chi-square $\left(\Sigma\left(y-y_{i}\right)^{2} / \sigma_{i}^{2}\right)$ where $y$ is a 
fitted value for a given point, $y_{i}$ is the measured data value for the point and $\sigma_{i}$ is the standard deviation for $y_{i}$. The errors in $I_{\lambda, T_{x}}^{a b s o r b e r}$ for any given temperature and wavelength were the combination of the statistical errors in $S_{\lambda, T_{x}}^{\text {absorber }}$ and the errors in the determination of the transfer function. The errors in the transfer functions were estimated by the variation of the transfer function for different temperatures and were the dominant source of error for all temperatures. Igor automatically calculates the estimated error (standard deviation) for each of the coefficients in a curve fit. The $T_{P t-1 P}$ agree with $T_{T C}$ to within the uncertainties. The graybody assumption $\left(T_{b b-2 P}\right)$ leads to a significant overestimation of the temperature of the sample. An interesting point is that even when the temperature dependence of the emissivity is taken into account by using $T F_{\lambda}^{\text {absorber }}$, the two-parameter fit $\left(T_{P t-2 P}, A_{P t-2 P}\right)$ has significantly lower accuracy in the temperature.

In Fig. 6, we simulate the one and two-parameter fits by adding random noise to calculated blackbody irradiances at $939 \mathrm{~K}$ and $797 \mathrm{~K}$ (two of the thermocouple temperatures). Here the one-parameter fit $(\varepsilon=1)$ results in temperature values that are in reasonable agreement with the temperature for the curve without noise, whereas in the two-parameter fit, the fitting function can adjust both the amplitude and temperature to generate a fit that is closer to all data points. Note that in this example the lower temperature data points have a higher two-parameter fit temperature than the higher temperature data points.

\section{Summary and Conclusion}

If the emissivity of a radiant emitter is unknown in optical pyrometry, greater accuracy can be achieved by fitting to a graybody curve in the short wavelength exponential region of the Planck irradiance curve. Not accounting for the wavelength and temperature dependence of the 
emissivity can lead to serious errors in the temperature determination. Lacking knowledge of the emissivity of a material used for temperature measurement, it can be absorbed into the optical transfer function of the system by a modified spectral calibration method. This method can be useful for applications such as remote temperature determination in an LHDAC where the wavelength dependence of the absorbing material may not be known.

\section{Acknowledgement}

Support for this research was provided by the NSF grant DMR-0804378, as well as a special grant from Seychelles Footwear and Alby and Kim Silvera. 


\section{References}

[1] N. Shekar, P. Sahu, and K. Rajan, J. of Mat. Sci. and Tech. 19, 518 (2003).

[2] N. Subramanian, N. Shaker, N. Kumar, and P. Sahu, Current Sci. 91 (2006).

[3] S. Meenakshi, V. Vijayakumar, and B. Godwal, Ind. Journ. of Pure and Appl. Phys. 44, 440 (2006).

[4] R. Boehler, Rev. Geophys. 38, 221 (2000).

[5] K. K. T. Walter M. J., Physics of the Earth and Planetary Interiors 143-144, 541 (2004).

[6] Y. Meng, G. Shen, and H. Mao, J. Phys. Cond. Matter. 18, 1097 (2006).

[7] S. Deemyad, E. Sterer, C. Barthel, S. Rekhi, J. Tempere, and I. F. Silvera, Rev. Sci. Instruments 76, 125104 (2005).

[8] J. Lin, W. Sturhahn, J. Zhao, G. Shen, H.-K. Mao, and R. J. Hemley, Geophys. Res. Lett. 31, L14611 (2004).

[9] G. Shen, H. K. Mao, and R. J. Hemley, Geophysical Res. Letters 25, 373 (1998).

[10] L. Ming, and W. A. Bassett, Rev. Sci. Inst. 45, 1115 (1974).

[11] A. P. Jephcoat, and S. P. Besedin, Phil. Trans. R. Soc. Lond. A 354, 1333 (1996).

[12] S. Rekhi, J. Tempere, and I. F. Silvera, Rev. Sci. Inst. 74, 3820 (2003).

[13] We approximate the emissivity with a linear function. In the long wavelength limit (Hagen-Rubens limit) the emissivity decreases as the square root of the frequency of light.

[14] P. Shuker, A. Melchior, Y. Assor, D. Belker, and E. Sterer, Rev. Sci. Inst. 79, 073908 (2008).

[15] L. S. Dubrovinsky, and S. K. Saxena, High-temperature High-Pressure 31, 393 (1999). 
[16] S. Deemyad, and I. F. Silvera, Rev. Sci. Inst. 79, 086105 (2008).

Table I. The best graybody ( $\varepsilon=$ const.) fit values to a non-blackbody irradiance at $\mathrm{T}=1000 \mathrm{~K}$ calculated at several $0.5 \mu \mathrm{m}$ intervals between $0.5-5 \mu \mathrm{m}$. The irradiance was calculated with $\varepsilon=$ $0.33-0.05 \lambda$ and these curves were fit with the graybody approximation.

$\begin{array}{llll}\text { Wavelength range } & \mathrm{T}_{\mathrm{GB}-2 \mathrm{P}}(\mathrm{K}) & \text { \% error in } \mathrm{T} & \varepsilon_{\mathrm{GB}-2 \mathrm{P}} \\ 0.5-1 \mu \mathrm{m} & 1010 & 1 & 0.25 \\ 1-1.5 \mu \mathrm{m} & 1022 & 2.2 & 0.21 \\ 1.5-2 \mu \mathrm{m} & 1043 & 4.3 & 0.18 \\ 2.5-3 \mu \mathrm{m} & 1079 & 7.9 & 0.14 \\ 3-3.5 \mu \mathrm{m} & 1142 & 11.4 & 0.11 \\ 3.5-4 \mu \mathrm{m} & 1252 & 12.5 & 0.07 \\ 4-4.5 \mu \mathrm{m} & \text { Non convergent } & & ------- \\ 4.5-5 \mu \mathrm{m} & \text { Non convergent } & & -------\end{array}$




\section{Table II}

Measured and calculated temperatures of the Pt source. $\mathrm{T}_{\mathrm{TC}}$ is the temperature of the Pt source measured by a thermocouple. $\mathrm{T}_{\mathrm{Pt} \_1 \mathrm{P}}$ is the temperature of the Pt calculated by the emissivity eliminated method with a one parameter fit $(\mathrm{T}) . \mathrm{T}_{\mathrm{Pt} \_2 \mathrm{P}}$ and $\mathrm{A}_{\mathrm{Pt}-2 \mathrm{P}}$ are the temperature and scaling coefficients calculated by the emissivity eliminated method with two fitting parameters, and $\mathrm{T}_{\mathrm{bb} \_2 \mathrm{P}}$ and $\varepsilon_{\mathrm{bb}-2 \mathrm{P}}$ are the temperature and the emissivity of the Pt calculated by the conventional correction of the signal by $T F_{\lambda, T}^{b b}$, assuming graybody behavior. All temperatures are in degrees Kelvin.

$\begin{array}{llllll}\mathrm{T}_{\mathrm{TC}} & \mathrm{T}_{\mathrm{Pt} \_1 \mathrm{P}} & \mathrm{T}_{\mathrm{Pt} \_2 \mathrm{P}} & \mathrm{A}_{\mathrm{Pt}-2 \mathrm{P}} & \mathrm{T}_{\mathrm{bb} \_2 \mathrm{P}} & \varepsilon_{\mathrm{bb}-2 \mathrm{P}} \\ 625 \pm 10 & 623 \pm 2.8 & 643 \pm 22 & 0.78 \pm 0.2 & 736 \pm 29 & 0.04 \pm 0.01 \\ 739 \pm 10 & 734 \pm 2.4 & 749 \pm 13 & 0.84 \pm 0.1 & 878 \pm 18 & 0.043 \pm 0.01 \\ 797 \pm 10 & 807 \pm 2.7 & 814 \pm 15 & 0.93 \pm 0.1 & 967 \pm 21 & 0.048 \pm 0.01 \\ 939 \pm 10 & 939 \pm 3.6 & 942 \pm 19 & 0.98 \pm 0.1 & 1154 \pm 29 & 0.05 \pm 0.01 \\ 1131 \pm 10 & 1130 \pm 5.1 & 1101 \pm 26 & 1.15 \pm 0.2 & 1408 \pm 44 & 0.06 \pm 0.01\end{array}$




\section{Figure Captions}

Figure 1. Irradiance of Pt (solid curve) and a blackbody source (dashed dot curve) at $1000 \mathrm{~K}$ calculated using the emissivity of platinum (dotted curve) $[15,16]$. The blackbody irradiance is scaled down by 0.16 in the graph. The dashed line is a two-parameter fit, assuming graybody behavior for the Pt.

Figure 2. Determination of the temperature for fitting to a GB in two different spectral regions using the emissivity function given in Table I.

Figure 3. Schematic drawing of the optical configuration for measuring temperature of an absorber and a blackbody in the infrared.

Figure 4. Corrected Pt thermal spectra: a) correction of the emissivity using Pt for the transfer function (inset graph is $T F_{\lambda, T}^{a b s o r b e r}$ and $T F_{\lambda, T}^{b b}$ of the optical system as a function of wavelength); b) Same, without emissivity correction, using a BB source for the transfer function. Thermocouple temperatures for different curves are shown for both a and $b$. Fit temperatures are tabulated in Table 1.

Fig. 5 Temperature of Pt determined by different methods listed in Table I as a function of temperature of Pt measured directly by a thermocouple. The dash-dot line represents the temperature equal to the thermocouple temperature. The 1-parameter fit has a slope close to 1 .

Fig. 6. Data generated by adding random noise to blackbody irradiance at $\mathrm{T}=939 \mathrm{~K}$ and $\mathrm{T}=797$ K. Solid curves are one parameter fits to data points and dashed curves are twoparameter fits. 


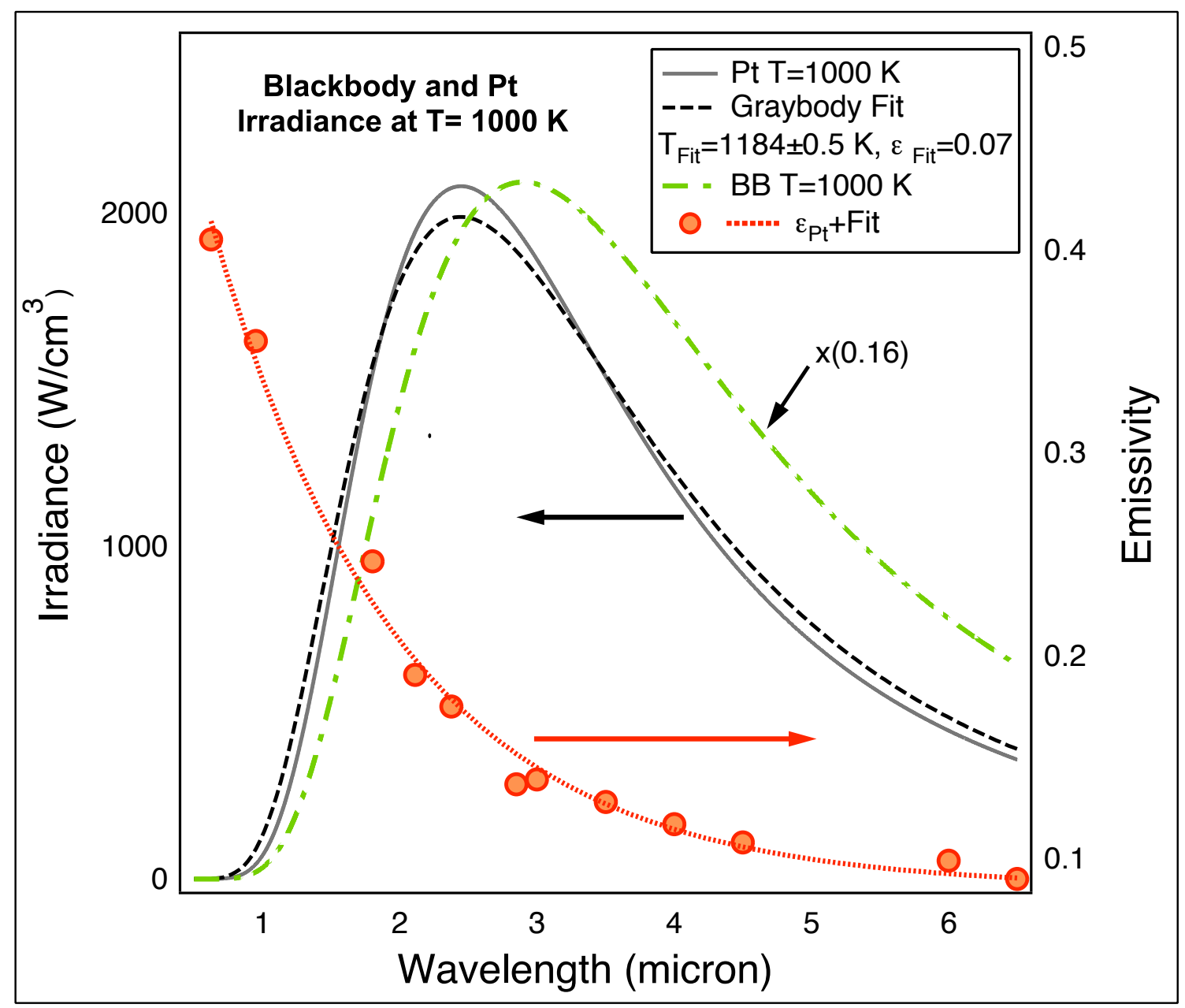

Figure 1. 

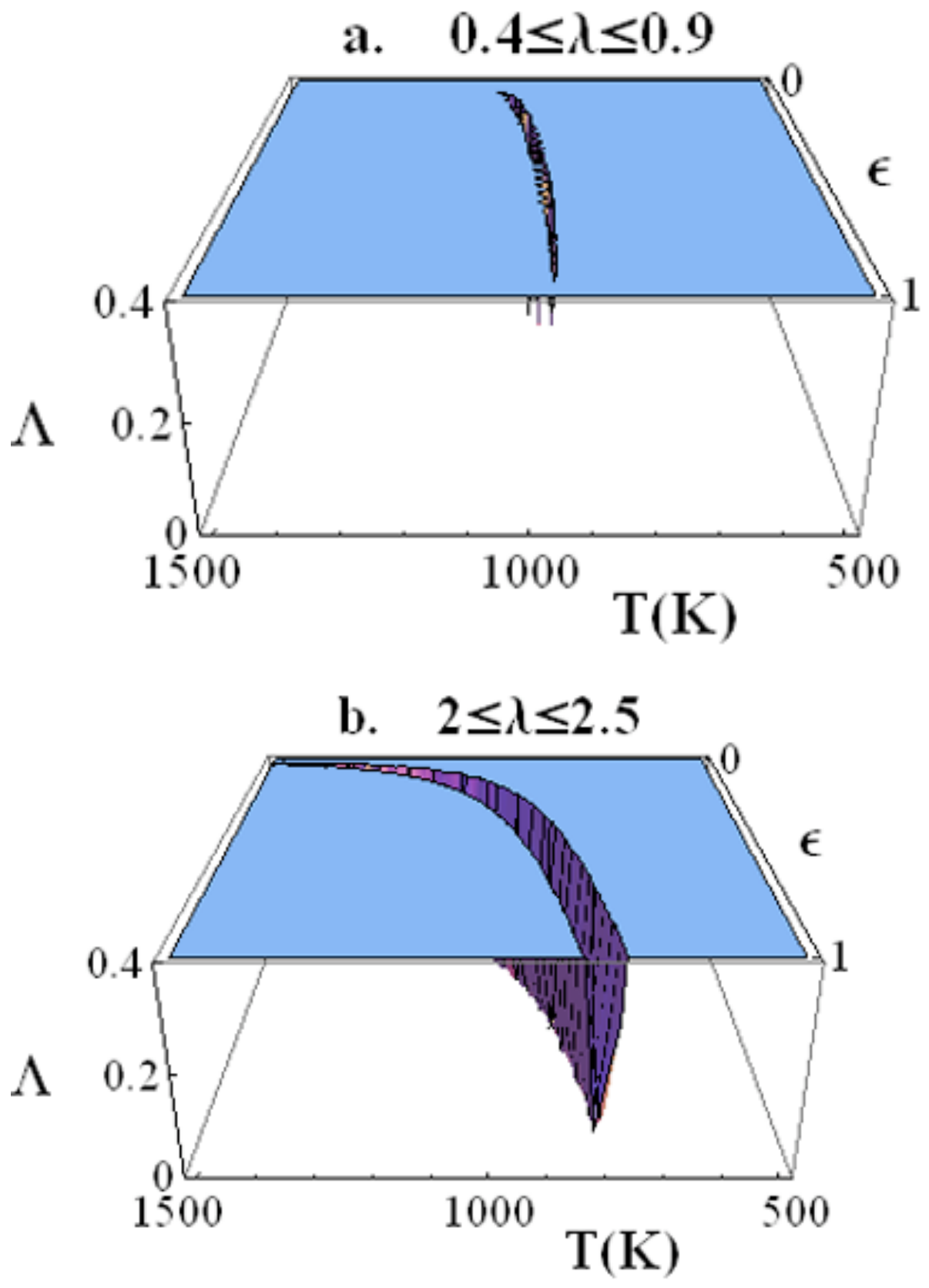

Figure. 2. 


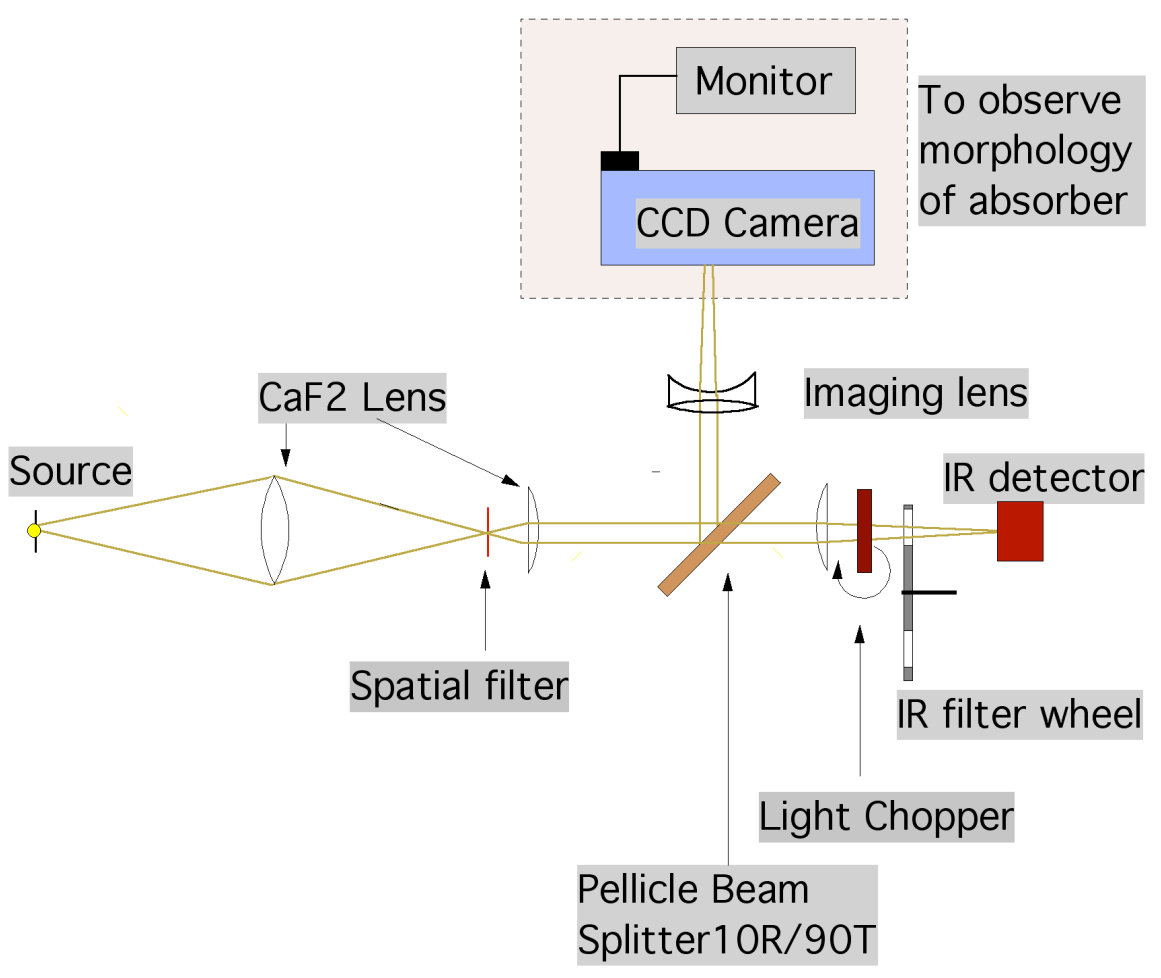

Figure 3. 

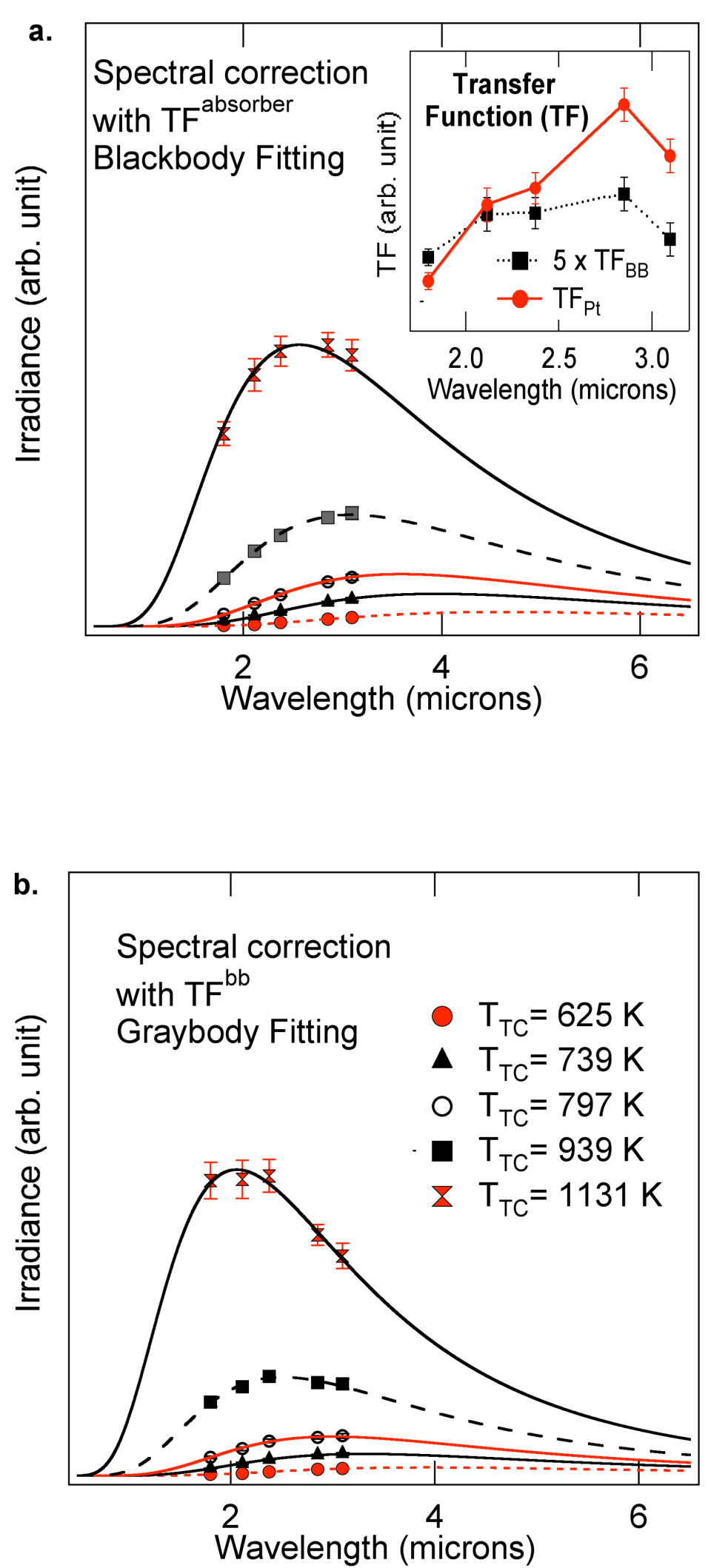

Figure 4. 


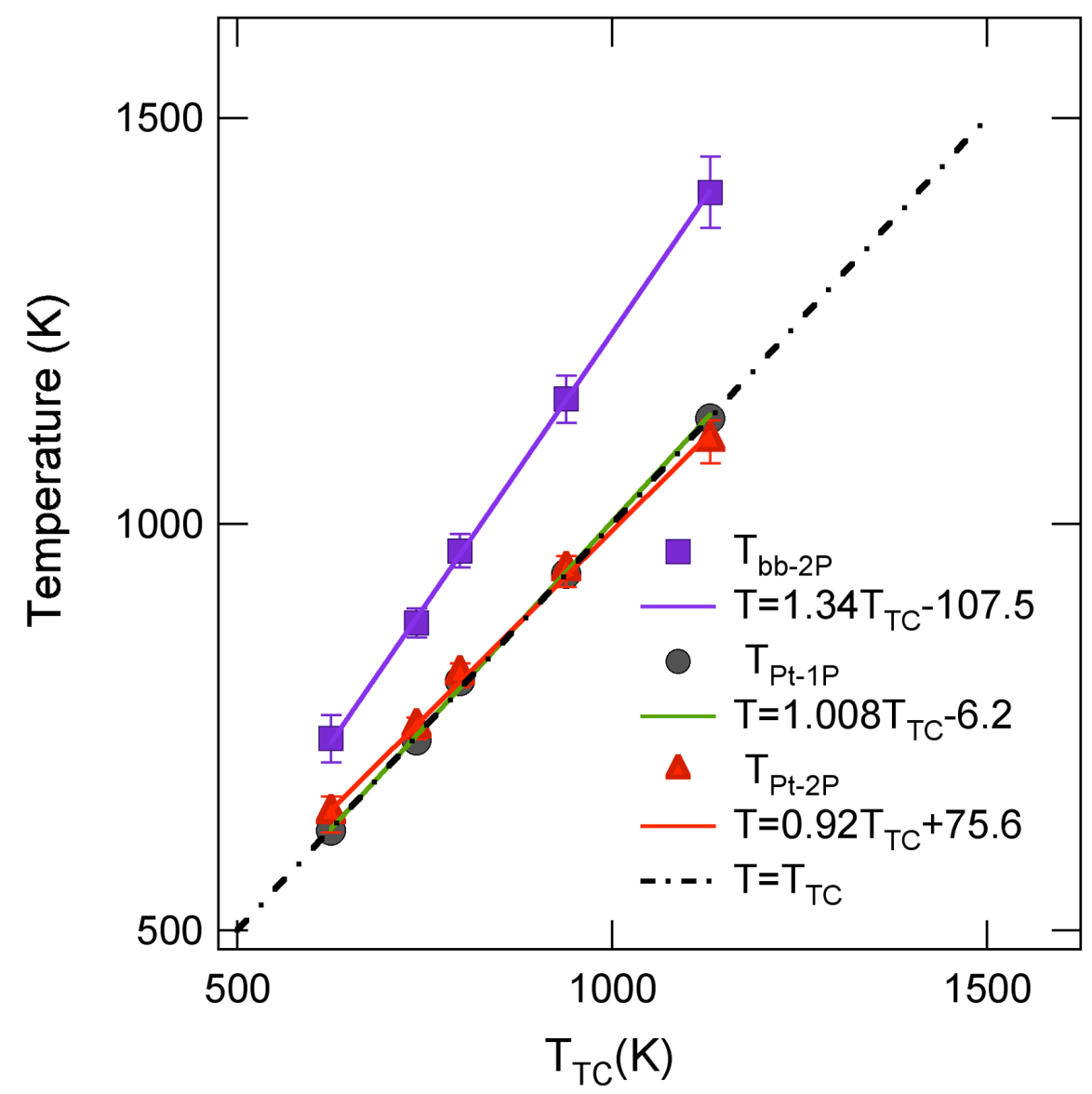

Figure 5. 


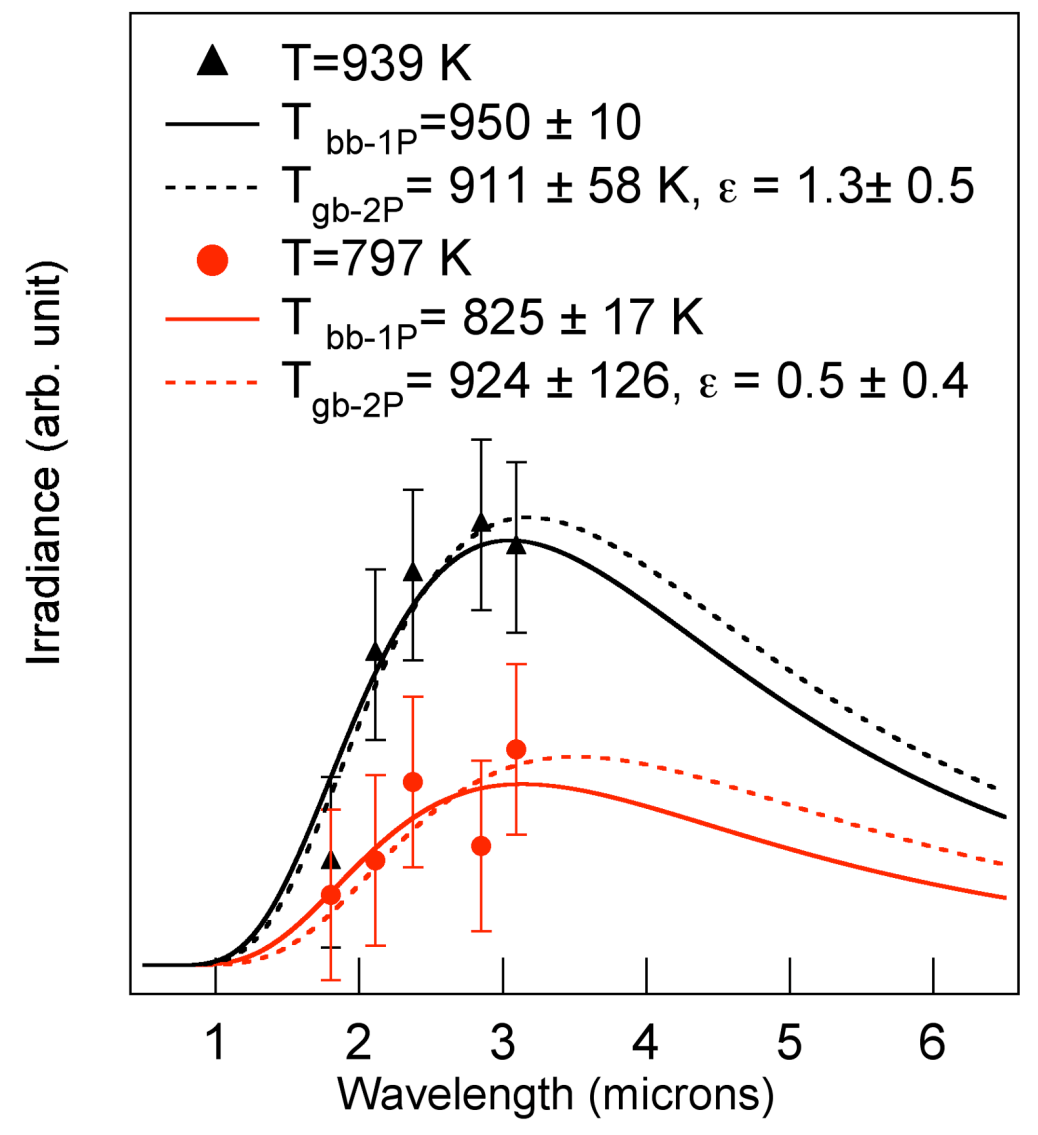

Figure 6 